\title{
A COVERING COCYCLE WHICH DOES NOT GROW LINEARLY
}

\author{
KATHLEEN M. MADDEN
}

\begin{abstract}
A cocycle $h: X \times Z^{m} \rightarrow R^{n}$ of a $Z^{m}$ action on a compact metric space, provides an $R^{n}$ suspension flow (analogous to a flow under a function) on a space $X_{h}$ which may not be Hausdorff or even $T_{1}$. Linear growth of $h$ guarantees that $X_{h}$ is a Hausdorff space; when $m=n$, linear growth is a consequence of $X_{h}$ being Hausdorff and a covering condition. This paper contains the construction of a cocycle $h: X \times Z \rightarrow R^{2}$ which does not grow linearly yet produces a locally compact Hausdorff space with the covering condition. The $Z$ action used in the construction is a substitution minimal set.
\end{abstract}

\section{INTRODUCTION}

Let $X$ be a compact metric space, and let $Z^{m}$ act as a group of commuting homeomorphisms on $X$. That is, we have a flow $\left(X, Z^{m}\right)$. For $a \in Z^{m}$, we denote the action of $a$ on $x \in X$ by $a x$. A cocycle for the flow $\left(X, Z^{m}\right)$ is a continuous map $h: X \times Z^{m} \rightarrow R^{n}$ satisfying the cocycle equation: for any $a, b \in Z^{m}$ and $x \in X$,

$$
h(x, a+b)=h(x, a)+h(a x, b) .
$$

A cocycle $h: X \times Z^{m} \rightarrow R^{n}$ can be used to construct the suspension $\left(X_{h}, R^{n}\right)$ of the flow $\left(X, Z^{m}\right)$. This is done as follows: we have a $Z^{m}$ action on $X \times R^{n}$ given by

$$
T_{a}(x, w)=(a x, w-h(x, a))
$$

for $a \in Z^{m}, x \in X, w \in R^{n}$. Because $h$ is a cocycle, it is easily checked that $T_{a} \circ T_{b}=T_{a+b}$ and hence $a \rightarrow T_{a}$ defines a $Z^{m}$ action on $X \times R^{n}$. We also have a natural $R^{n}$ action on $X \times R^{n}$ via

$$
((x, v), w) \rightarrow(x, v+w)
$$

for $x \in X, v, w \in R^{n}$. These two actions commute and so the $R^{n}$ flow on $X \times R^{n}$ gives an $R^{n}$ action on $X_{h}$, where $X_{h}$ is the quotient space of $X \times R^{n}$ modulo the $Z^{m}$ action on it. When $m=n=1$, this construction yields the familiar flow under a function.

Received by the editors April 4, 1994; originally communicated to the Proceedings of the AMS by Linda Keen.

1991 Mathematics Subject Classification. Primary 58F25; Secondary 28D10, 54H20.

Research was supported in part by NSF Grant \#01524638. 
In the hope of being able to use suspensions to create interesting $R^{n}$ flows, we would like to insist that $X_{h}$ is Hausdorff and $\left(X_{h}, R^{n}\right)$ is minimal. It is not hard to check that $\left(X_{h}, R^{n}\right)$ will be minimal if and only if $\left(X, Z^{m}\right)$ is minimal.

The relationship between the properties of $h$ and the topological properties of the resulting suspension $\left(X_{h}, R^{n}\right)$ has been studied by Furstenberg, Keynes, Markley, and Sears in [3].

Definition 1.1. A cocycle $h$ is covering if

(i) $X_{h}$ is a Hausdorff space,

(ii) the projection $\pi: X \times R^{n} \rightarrow X_{h}$ is a local homeomorphism.

When $h$ is covering, $X_{h}$ is locally compact and metric. One of the goals of [3] was to gain a basic understanding of covering cocycles. Some of the results are included here under the assumption that the $Z^{m}$ action on $X$ has a free dense orbit.

Theorem 1.1. A cocycle $h: X \times Z^{m} \rightarrow R^{n}$ is covering if and only if $|h(x, a)| \rightarrow$ $\infty$ uniformly in $x$ as $|a| \rightarrow \infty$.

(The norm we will use is $|v|=\sum\left|v_{i}\right|$. )

Theorem 1.2. Let $h: X \times Z^{m} \rightarrow R^{n}$ be a covering cocycle. Then $n \geq m$, and the space $X_{h}$ is compact if and only if $n=m$.

The key result needed to show that $m=n$ when $X_{h}$ is compact is:

Theorem 1.3. If $h$ is a covering cocycle and $X_{h}$ is compact, then there exist constants $C$ and $D$ such that

$$
C|a| \leq|h(x, a)|
$$

whenever $|a| \geq D$.

In fact, as a consequence of the cocycle equation, given any cocycle $h: X \times Z^{m} \rightarrow R^{n}$, there exists a constant $C^{\prime}>0$ such that $|h(x, a)| \leq C^{\prime}|a|$ for all $a \in Z^{m}$. Thus, for $h$ as in Theorem 1.3,

$$
C|a| \leq|h(x, a)| \leq C^{\prime}|a|
$$

when $|a|$ is sufficiently large.

These results lead to the question of whether the covering cocycles are precisely the cocycles which grow linearly in the sense of Theorem 1.3. Clearly, when $n=m$ the answer is yes. In what follows we will show that when $n \neq m$, covering and linear growth are not equivalent notions. We will construct a covering cocycle on a minimal flow which does not grow linearly.

Our example is obtained using a substitution minimal flow $(\overline{\mathscr{O}(\omega)}, \sigma)$ and a cocycle $h: \overline{\mathscr{O}(\omega)} \times Z \rightarrow R^{2}$. In section 2 we discuss some general properties of substitution minimal flows and cocycles on such flows. In section 3 we construct the specific substitution minimal flow and cocycle $h$ on it which is covering but does not grow linearly. 


\section{SubSTitution MiNimal flows}

Let $(X, T)$ be a flow (i.e., $T: X \rightarrow X$ is a homeomorphism of $X$ onto $X$ ), and suppose that $\omega \in X$ has a free dense orbit. If $h: X \times Z \rightarrow R^{n}$ is a cocycle for the flow $(X, T)$, we may characterize when $h$ is covering in terms of $\omega$ :

Proposition 2.1. Let $(X, T), \omega \in X$, and $h$ be as described. Then $h$ is covering if and only if given $R>0$, there exists $r>0$ such that $|a-b|>r$ implies $|h(\omega, a)-h(\omega, b)|>R$.

Proof. First suppose $h$ is covering and let $R>0$ be given. We can choose $r>0$ such that $|a|>r$ implies $|h(x, a)|>R$ for all $x \in X$. In particular, using the cocycle equation,

$$
|h(\omega, a)-h(\omega, b)|=\left|h\left(T^{b}(\omega), a-b\right)\right|>R
$$

when $|a-b|>r$.

Conversely, let $R>0$ be given and choose $r>0$ as described. Now suppose $x \in X$ and $a \in Z$ with $|a|>r$. For some $\left\{n_{i}\right\}_{i=0}^{\infty} \subseteq Z, T^{n_{i}}(\omega) \rightarrow x$ and

$$
\begin{aligned}
|h(x, a)| & =\lim _{i \rightarrow \infty}\left|h\left(T^{n_{i}}(\omega), a\right)\right| \\
& =\lim _{i \rightarrow \infty}\left|h\left(\omega, a+n_{i}\right)-h\left(\omega, n_{i}\right)\right| \\
& \geq R .
\end{aligned}
$$

Thus, $|h(x, a)| \rightarrow \infty$ uniformly in $x \in X$ as $|a| \rightarrow \infty$.

We also observe that for $(X, T)$ a flow, there is a one-to-one correspondence between $\mathscr{C}=\left\{h: X \times Z \rightarrow R^{n} \mid h\right.$ is a cocycle $\}$ and $C\left(X, R^{n}\right)=\left\{f: X \rightarrow R^{n} \mid\right.$ $f$ is continuous $\}$. For $f \in C\left(X, R^{n}\right)$ we obtain $h_{f} \in \mathscr{C}$ as follows:

$$
h_{f}(x, n)= \begin{cases}\sum_{i=0}^{n-1} f\left(T^{i} x\right), & n>0, \\ 0, & n=0, \\ -\sum_{i=1}^{-n} f\left(T^{-i} x\right), & n<0 .\end{cases}
$$

Conversely, if $h \in \mathscr{C}$, then $h(\cdot, 1): X \rightarrow R^{n}$ is in $C\left(X, R^{n}\right)$.

In particular, for $S=\{1,2, \ldots, m\}$ a finite collection of symbols and $\left(S^{Z}, \sigma\right)$ the usual full shift on $m$ symbols, $\left(S^{Z}, \sigma\right)$ is a flow. By choosing a non-periodic point $\omega \in S^{Z}$, we can construct $(\overline{\mathscr{O}(\omega)}, \sigma)=(X, \sigma)$ where $\overline{\mathscr{O}(\omega)}=\overline{\left\{\sigma^{k}(\omega): k \in Z\right\}}$. Then $(X, \sigma)$ is a flow with $\omega \in X$ having a free dense orbit. We obtain a collection of cocycles on $(X, \sigma)$ by considering maps from $S$ into $R^{n}$. If $f: S \rightarrow R^{n}$, then for $\tilde{f}: X \rightarrow R^{n}$ via $\tilde{f}(x)=f\left(x_{0}\right), \tilde{f}$ is in $C\left(X, R^{n}\right)$ and gives $h=h_{\tilde{f}}$ in $\mathscr{C}$ as described. (We may extend $f$ to finite blocks of symbols by $f\left(s_{1} \ldots s_{k}\right)=\sum_{i=1}^{k} f\left(s_{i}\right)$. Then $h(x, n)=f(x[0, n-1])$ for $n>0$ and $h(x, n)=-f(x[n,-1])$ for $n<0$.) Under these circumstances and the additional assumption that $(X, \sigma)$ is uniquely ergodic, the following proposition illustrates that the linear growth of $h$ and the values taken on by $f$ are closely connected.

Proposition 2.2. Let $(X, \sigma), f$, and $h$ be as described. Suppose also that $(X, \sigma)$ is uniquely ergodic with unique invariant measure $\phi$. Then the following are equivalent: 
(1) There exists $r, R, R^{\prime}>0$ such that $|a|>r$ implies

$$
R^{\prime}|a| \leq|h(x, a)| \leq R|a|
$$

for all $x \in X$

(2) There exists $r, R, R^{\prime}>0$ such that $|a-b|>r$ implies

$$
R^{\prime}|a-b| \leq|h(\omega, a)-h(\omega, b)| \leq R|a-b|
$$

(3) $\left|\sum_{s \in S} f(s) \phi(s)\right|>0$.

Proof. It is clear that (1) implies (2) since

$$
|h(\omega, a)-h(\omega, b)|=\left|h\left(\sigma^{b}(\omega), a-b\right)\right| .
$$

Suppose that (2) holds. Let $x \in X$ with $\sigma^{n_{i}}(\omega) \rightarrow x$. Then

$$
|h(x, a)|=\lim _{i \rightarrow \infty}\left|h\left(\sigma^{n_{i}}(\omega), a\right)\right|=\lim _{i \rightarrow \infty}\left|h\left(\omega, a+n_{i}\right)-h\left(\omega, n_{i}\right)\right|,
$$

and it is clear that (2) implies (1).

Now suppose that $a, b \in Z, a<b$. We see

$$
|h(\omega, a)-h(\omega, b)|=\left|\sum_{s \in S} f(s) N_{s}(\omega[a, b-1])\right|,
$$

where $N_{s}(\omega[a, b-1])$ denotes the number of occurrences of the symbol $s$ in the block $\omega[a, b-1]$. In particular, for any $a \in Z$, by the unique ergodicity of $(X, \sigma)$

$$
\begin{aligned}
\lim _{m \rightarrow \infty}\left|\frac{h(\omega, a)-h(\omega, a+m)}{m}\right| & =\lim _{m \rightarrow \infty}\left|\sum_{s \in S} f(s) \frac{N_{s}(\omega[a, a+m-1])}{m}\right| \\
& =\left|\int \tilde{f} d \phi\right|=\left|\sum_{s \in S} f(s) \phi(s)\right| .
\end{aligned}
$$

Since this limit is uniform in $a \in Z$, it is apparent that (2) holds if and only if (3) holds.

Notice that because the constant $R$ in (1) exists for any cocycle, if (3) fails to hold then (1) fails because $R^{\prime}$ does not exist.

With these results in mind, we turn to substituting minimal flows as a way of constructing uniquely ergodic, minimal flows $(X, \sigma)$ with $X=\overline{\mathscr{O}(\omega)} \subseteq S^{Z}$. A substitution is a map sending symbols from a finite set into finite strings of these symbols, called blocks. A thorough overview of substitutions can be found in [1] and [8]. For completeness, we include some of the notation and results here.

For $S=\{1,2, \ldots, m\}$ as before, let $S^{n}$ denote the collection of blocks of length $n$. Then $\theta: S \rightarrow S^{n}$ is a substitution of constant length. We associate an $m \times m$ matrix, $M_{\theta}$, with $\theta$ via

$$
M_{\theta}=\left(N_{i}(\theta(j))\right)_{1 \leq i, j \leq m}
$$

where $N_{i}(\theta(j))$ is the number of occurrences of the symbol $i$ in the block $\theta(j)$. We say $\theta$ is primitive if the matrix $M_{\theta}$ is primitive in the usual sense. 
Let $\theta$ be a primitive substitution. We say a pair of symbols $p, q \in S$ is a recurrent pair if

(i) $\theta(q)$ begins with $q$ and $\theta(p)$ ends with $p$,

(ii) the block $p q$ occurs in $\theta^{k}(q)$ for some $k \geq 1$.

A recurrent pair of symbols determines an element of $S^{Z}$, denoted $\omega^{p q}=\omega$, by

$$
\omega\left[-n^{k}, n^{k}-1\right]=\theta^{k}(p q)
$$

where $\omega[i, i+j]=\omega_{i} \omega_{i+1} \ldots \omega_{i+j}$. Notice that $\theta(\omega)=\omega$. Then $\overline{\mathscr{O}(\omega)}=$ $\overline{\left\{\sigma^{k}(\omega): k \in Z\right\}}$ is an invariant subset of $S^{Z}$. In fact we can say more:

Theorem 2.1. If $\theta$ is a substitution with a recurrent pair $p q$ and $\omega=\omega^{p q} \in S^{Z}$, then $(\overline{\mathscr{O}(\omega)}, \sigma)$ is minimal and uniquely ergodic.

The minimality of $(\overline{\mathscr{O}(\omega)}, \sigma)$ was first observed by Gottschalk [4] and the unique ergodicity by Klein [5]. Others, including Dekking [1] and Michel [7], have extended these results to more general situations than those discussed here.

If $\phi$ is the unique invariant measure on $\overline{\mathscr{O}(\omega)}$, then $(\phi(s))_{s \in S}$ is the normalization of the strictly positive right eigenvector associated with the PerronFrobenius eigenvalue of $M_{\theta}$ [7].

\section{A COVERING COCYCLE WHICH DOES NOT GROW LINEARLY}

Let $S=\{a, b, c, d, \hat{a}, \hat{b}, \hat{c}, \hat{d}\}$ and let $\theta: S \rightarrow S^{4}$ as follows:

$$
\theta(a)=a b c a, \theta(b)=b d a b, \theta(c)=c a \hat{d} c, \theta(d)=d \hat{c} b d,
$$

and $\theta(\hat{s})=\hat{s} \hat{s_{1}} \hat{s_{2}} \hat{s}$ for $\theta(s)=s s_{1} s_{2} s$ and $\hat{\hat{s}}=s$.

We see that

$$
M_{\theta}=\left(\begin{array}{llllllll}
2 & 1 & 1 & 0 & 0 & 0 & 0 & 0 \\
1 & 2 & 0 & 1 & 0 & 0 & 0 & 0 \\
1 & 0 & 2 & 0 & 0 & 0 & 0 & 1 \\
0 & 1 & 0 & 2 & 0 & 0 & 1 & 0 \\
0 & 0 & 0 & 0 & 2 & 1 & 1 & 0 \\
0 & 0 & 0 & 0 & 1 & 2 & 0 & 1 \\
0 & 0 & 0 & 1 & 1 & 0 & 2 & 0 \\
0 & 0 & 1 & 0 & 0 & 1 & 0 & 2
\end{array}\right),
$$

and it is easily verified that $M_{\theta}$ is primitive and that $c a$ is a recurrent pair. Thus, for $\omega=\omega^{c a},(\overline{\mathscr{\sigma}(\omega)}, \sigma)=\left(X_{\omega}, \sigma\right)$ is minimal and uniquely ergodic. Let $\phi$ be the unique invariant measure on $\left(X_{\omega}, \sigma\right)$. Since the Perron-Frobenius eigenvalue of $M_{\theta}$ is 4 and the corresponding right eigenvector is $(1,1, \ldots, 1)$, $\phi(s)=1 / 8$ for all $s \in S$.

Let $f: S \rightarrow R^{2}$ by

$$
\begin{array}{ll}
f(a)=(\sqrt{2}, 0), & f(b)=(1,1), \\
f(c)=(1,-1), & f(d)=(0, \sqrt{2})
\end{array}
$$

and $f(\hat{s})=-f(s)$. Then, as described in the previous section, we have $\tilde{f} \in$ $C\left(X_{\omega}, R^{2}\right)$ and $h=h_{\tilde{f}} \in \mathscr{C}=\left\{g: X_{\omega} \times Z \rightarrow R^{2} \mid g\right.$ is a cocycle $\}$. By Proposition 2.2, $h$ does not grow linearly since $\left|\sum_{s \in S} f(s) \phi(s)\right|=0$. Also, 
if we let $\tilde{f}(x)=\left(\tilde{f}_{1}(x), \tilde{f}_{2}(x)\right)$, then $\int_{X_{\omega}} \tilde{f}_{i} d \phi=0$ for $i=1,2$ and so, by Theorem 1.12 in [3], neither $\tilde{f}_{1}$ nor $\tilde{f}_{2}$ would determine a covering cocycle on $X_{\omega}$. In particular, trying to build a flow under either $\tilde{f}_{1}$ or $\tilde{f}_{2}$ would be fruitless. However, in what follows we will show that taken in tandem, they produce $h$, a covering cocycle with values in $R^{2}$.

We first note that for

$$
L=\left(\begin{array}{cc}
2+\sqrt{2} & 0 \\
0 & 2+\sqrt{2}
\end{array}\right)
$$

$L f=f \theta$ and, by induction, $L^{n} f=f \theta^{n}$. Denote $2+\sqrt{2}$ by $\alpha$. (The substitution $\theta$ and the matrix $L$ are variants of a substitution and matrix used by Dekking in [2] to create a continuous nowhere differentiable curve, the von Koch curve. Dekking uses the substitution to construct compact subsets $\left\{A_{n}\right\}_{n=1}^{\infty}$ of $R^{2}$; his compact sets, $A_{n}$, resemble the image of the first $4^{n}$ integers (connected with line segments) under our $h(\omega, \cdot)$. Dekking then uses $L^{-1}$ as a scaling transformation to obtain the von Koch curve as the limit of the sets $L^{-n} A_{n}$.)

To show that $h$ is covering, by Proposition 2.1 it will suffice to show that given $R>0$ there exists $r>0$ so that when $|n-m|>r$ for $n, m \in Z$ (without loss of generality $n<m$ ), then

$$
|h(\omega, n)-h(\omega, m)|=|f(\omega[n, m-1])|>R .
$$

The following three lemmas will be used.

Lemma 3.1. Let $W$ be a block appearing in $\omega$, and let $N(W)$ denote the number of symbols in $W$. Then for all $n \geq 0$,

(i) $N(W)=1$ implies $\sqrt{2} \alpha^{n} \leq\left|f \theta^{n}(W)\right| \leq 2 \alpha^{n}$,

(ii) $N(W)=2$ implies $2 \alpha^{n} \leq\left|f \theta^{n}(W)\right| \leq \alpha^{n+1}$,

(iii) $N(W)=3$ implies $\alpha^{n+1} \leq\left|f \theta^{n}(W)\right| \leq \sqrt{2} \alpha^{n+1}$,

(iv) $N(W)=4$ implies $\sqrt{2} \alpha^{n+1} \leq\left|f \theta^{n}(W)\right| \leq 2 \alpha^{n+1}$,

(v) $N(W)=5$ implies $\sqrt{2} \alpha^{n+1} \leq\left|f \theta^{n}(W)\right|$.

Proof. We observe that it suffices to show that the inequalities hold for $n=0$ since

$$
\left|f \theta^{n}(W)\right|=\left|L^{n} f(W)\right|=\alpha^{n}|f(W)| .
$$

To show that the lemma holds for $n=0$, we list all the blocks of length 5 or less appearing in $\omega$ and we apply $f$ to all of these blocks.

This is not as tedious as it might seem. There are several simplifying assumptions we can make. First, it can be shown that each symbol can only be followed by two others. For example, $a$ can be followed by $b$ and $\hat{d}$. Secondly, if $s_{1} \ldots s_{n}$ appears in $\omega$ for $1 \leq n \leq 5$, then we must be able to find $t_{1} t_{2}$ appearing in $\omega$ with $s_{1} \ldots s_{n}$ a subblock of $\theta\left(t_{1} t_{2}\right)$. These two observations considerably shorten the list of possible words of length 5 or less appearing in $\omega$. Also the fact that $\left|f\left(s_{1} \ldots s_{n}\right)\right|=\left|-f\left(\hat{s_{1}} \ldots \hat{s_{n}}\right)\right|$ reduces the necessary calculations further. These calculations can be found in Table 1 of [6] .

The remaining two lemmas give approximations for values of $f$ on blocks of the form

$$
\theta^{n-1}\left(s_{1} \ldots s_{i}\right) \theta^{n}\left(t_{1} \ldots t_{k}\right) \theta^{n-1}\left(u_{1} \ldots u_{j}\right)
$$


$0 \leq i, j \leq 3,1 \leq k \leq 4$, when they occur in $\omega$ in a particular location. The word "occurs" will be given a special technical meaning; specifically, when we say a block of this form occurs in $\omega$, we mean it appears in the following way: $\theta\left(s_{i}\right)$ ends in $s_{1} \ldots s_{i}, \theta\left(u_{1}\right)$ begins with $u_{1} \ldots u_{j}$, and

$$
\omega\left[0, p \cdot 4^{n}-1\right]=\theta^{n}(a) \ldots \theta^{n}\left(s_{i}\right) \theta^{n}\left(t_{1} \ldots t_{k}\right) \theta^{n}\left(u_{1}\right)
$$

where $\omega[0, p-1]$ begins with $a$ and ends with $s_{i} t_{1} \ldots t_{k} u_{1}$ or that

$$
\omega\left[-p \cdot 4^{n},-1\right]=\theta^{n}\left(s_{i}\right) \theta^{n}\left(t_{1} \ldots t_{k}\right) \theta^{n}\left(u_{1}\right) \ldots \theta^{n}(c)
$$

where $\omega[-p,-1]$ begins with $s_{i} t_{1} \ldots t_{k} u_{1}$ and ends with $c$. This distinction is important because not every appearance of a block of the above form is an occurrence.

Lemma 3.2. Suppose $0 \leq i \leq 3,2 \leq k \leq 4$.

(1) If $s_{1} \ldots s_{i} \theta\left(t_{1} \ldots t_{k}\right)$ occurs in $\omega$, then

$$
\left|f\left(s_{1} \ldots s_{i} \theta\left(t_{1} \ldots t_{k}\right)\right)\right| \geq\left|f\left(s_{1} \ldots s_{i}\right)\right|+\left|f\left(\theta t_{1}\right)\right| .
$$

(2) If $\theta\left(t_{1} \ldots t_{k}\right) s_{1} \ldots s_{i}$ occurs in $\omega$, then

$$
\left|f\left(\theta\left(t_{1} \ldots t_{k}\right) s_{1} \ldots s_{i}\right)\right| \geq\left|f\left(\theta t_{1}\right)\right|+\left|f\left(s_{1} \ldots s_{i}\right)\right| .
$$

Proof. If $i=0$, then the result follows from Lemma 3.1. The case of $k=2$ is handled by calculating $\left|f\left(s_{1} \ldots s_{i} \theta\left(t_{1} \ldots t_{k}\right)\right)\right|,\left|f\left(s_{1} \ldots s_{i}\right)\right|+\left|f\left(\theta t_{1}\right)\right|$, and $\left|f\left(\theta\left(t_{1} \ldots t_{k}\right) s_{1} \ldots s_{i}\right)\right|$ on all possible blocks of length three occurring in $\omega$. These calculations are contained in Table 2 of [6].

Otherwise, $k>2$. We will show that (1) holds. The proof of (2) is similar.

First, suppose that $i=1$. Then, using Lemma 3.1 twice,

$$
\begin{aligned}
\left|f\left(s_{1} \theta\left(t_{1} \ldots t_{k}\right)\right)\right| & \geq|| f\left(\theta\left(t_{1} \ldots t_{k}\right)\right)|-| f\left(s_{1}\right)|| \geq \alpha^{2}-2 \\
& \geq 2 \alpha+2 \geq\left|f\left(\theta t_{1}\right)\right|+\left|f\left(s_{1}\right)\right| .
\end{aligned}
$$

Next suppose that $i=2$ and suppose $\theta\left(s_{2}\right)=s_{2} s_{3} s_{1} s_{2}$. Then

$$
\begin{aligned}
\left|f\left(s_{1} s_{2} \theta\left(t_{1} \ldots t_{k}\right)\right)\right| & \geq|| f\left(\theta\left(s_{2} t_{1} \ldots t_{k}\right)\right)|-| f\left(s_{2} s_{3}\right)|| \\
& \geq \sqrt{2} \alpha^{2}-\alpha \geq 2 \alpha+\alpha \\
& \geq\left|f\left(\theta t_{1}\right)\right|+\left|f\left(s_{1} s_{2}\right)\right|
\end{aligned}
$$

A similar argument holds for $i=3$.

Lemma 3.3. Suppose $s_{1} \ldots s_{i} \theta\left(t_{1} \ldots t_{k}\right) u_{1} \ldots u_{j}$ occurs in $\omega$, with $0 \leq i, j \leq 3$ and $1 \leq k \leq 4$. Then, for all $n \in N$,

$$
\left|f\left[\theta^{n-1}\left(s_{1} \ldots s_{i}\right) \theta^{n}\left(t_{1} \ldots t_{k}\right) \theta^{n-1}\left(u_{1} \ldots u_{j}\right)\right]\right| \geq \sqrt{2} \alpha^{n}
$$


Proof. We observe that the proof reduces to showing that the inequality holds for $n=1$, because for $n>1$, we have the following easy inductive step:

$$
\begin{aligned}
\left|f\left(\theta^{n-1}\left(s_{1} \ldots s_{i}\right) \theta^{n}\left(t_{1} \ldots t_{k}\right) \theta^{n-1}\left(u_{1} \ldots u_{j}\right)\right)\right| \\
\quad=\left|L f\left(\theta^{n-2}\left(s_{1} \ldots s_{i}\right) \theta^{n-1}\left(t_{1} \ldots t_{k}\right) \theta^{n-2}\left(u_{1} \ldots u_{j}\right)\right)\right| \\
\quad=\alpha\left|f\left(\theta^{n-2}\left(s_{1} \ldots s_{i}\right) \theta^{n-1}\left(t_{1} \ldots t_{k}\right) \theta^{n-2}\left(u_{1} \ldots u_{j}\right)\right)\right| \\
\quad \geq \alpha\left(\sqrt{2} \alpha^{n-1}\right) \\
\quad=\sqrt{2} \alpha^{n} .
\end{aligned}
$$

First suppose that $k=1$. Lemma 3.1 includes $i=j=0$. If $j=3$ and $i>1$ then, using Lemma 3.1 and Lemma 3.2,

$$
\begin{aligned}
\left|f\left(s_{1} \ldots s_{i} \theta\left(t_{1}\right) u_{1} u_{2} u_{3}\right)\right| & \geq|| f\left(s_{1} \ldots s_{i} \theta\left(t_{1} u_{1}\right)\right)|-| f\left(u_{1}\right)|| \\
& \geq\left|f\left(\theta t_{1}\right)\right|+\left|f\left(s_{1} \ldots s_{i}\right)\right|-\left|f\left(u_{1}\right)\right| \\
& \geq\left|f\left(\theta t_{1}\right)\right| \\
& \geq \sqrt{2} \alpha .
\end{aligned}
$$

The case of $j>1$ and $i=3$ is similar. The remaining cases for $k=1$ are handled in Tables 3 and 4 of [6]. 3.2 ,

Otherwise, $k \geq 2$. Suppose $\left|f\left(s_{1} \ldots s_{i}\right)\right| \geq\left|f\left(u_{1} \ldots u_{j}\right)\right|$. Then, using Lemma

$$
\begin{aligned}
\left.\mid f\left(s_{1} \ldots s_{i} \theta\left(t_{1} \ldots t_{k}\right) u_{1} \ldots u_{j}\right)\right) \mid & \geq|| f\left(s_{1} \ldots s_{i} \theta\left(t_{1} \ldots t_{k}\right)\right)|-| f\left(u_{1} \ldots u_{j}\right)|| \\
& \geq\left|f\left(\theta t_{1}\right)\right|+\left|f\left(s_{1} \ldots s_{i}\right)\right|-\left|f\left(u_{1} \ldots u_{j}\right)\right| \\
& \geq\left|f\left(\theta t_{1}\right)\right| \\
& \geq \sqrt{2} \alpha .
\end{aligned}
$$

Similarly if $\left|f\left(u_{1} \ldots u_{j}\right)\right| \geq\left|f\left(s_{1} \ldots s_{i}\right)\right|$.

We are now ready to show that $h$ is covering.

Theorem 3.1. There exists $c>0$ such that when $4^{n}<|a-b| \leq 4^{n+1}$ then

$$
|h(\omega, a)-h(\omega, b)| \geq c \alpha^{n-1}
$$

for all $n \in N$ and $h$ is a covering cocycle.

Proof. Assuming $a<b$, first note that if

$$
W=s_{0_{1}} \ldots s_{0_{i_{0}}} \theta\left(s_{1_{1}} \ldots s_{1_{i_{1}}}\right) \theta^{2}\left(s_{2_{1}} \ldots s_{2_{i_{2}}}\right) \ldots \theta^{n-2}\left(s_{(n-2)_{1}} \ldots s_{(n-2)_{i_{(n-2)}}}\right)
$$

appears in $\omega$ with $0 \leq i_{j} \leq 3$ and $0 \leq j \leq n-2$, then, using Lemma 3.1, we have

$$
\begin{aligned}
|f(W)| & \leq \sum_{j=0}^{n-2} \mid f\left(\theta^{j}\left(s_{j_{1}} \ldots s_{j_{i_{j}}}\right) \mid \leq \sum_{j=0}^{n-2} \sqrt{2} \alpha^{j+1}\right. \\
& =\sqrt{2} \alpha\left(\frac{1-\alpha^{n-1}}{1-\alpha}\right)=\sqrt{2} \alpha\left(\frac{\alpha^{n-1}-1}{\alpha-1}\right) \leq \frac{\sqrt{2} \alpha^{n}}{\alpha-1} .
\end{aligned}
$$

We consider two possible cases. 
Case 1. A $\theta^{n}(s)$ block occurs in $\omega[a, b-1]$ for some $s \in S$. (Notice at most four such blocks can occur in $\omega[a, b-1]$.) So

$$
\begin{aligned}
& \omega[a, b-1]=s_{0_{1}} \ldots s_{0_{i_{0}}} \theta\left(s_{1_{1}} \ldots s_{1_{i_{1}}}\right) \\
& \quad \ldots \theta^{n-2}\left(s_{(n-2)_{1}} \ldots s_{(n-2)_{i_{(n-2)}}}\right) \theta^{n-1}\left(s_{1} \ldots s_{i}\right) \theta^{n}\left(t_{1} \ldots t_{k}\right) \theta^{n-1}\left(u_{1} \ldots u_{j}\right) \\
& \quad \ldots u_{0_{1}} \ldots u_{0_{j_{0}}}
\end{aligned}
$$

with $\theta^{n-1}\left(s_{1} \ldots s_{i}\right) \theta^{n}\left(t_{1} \ldots t_{k}\right) \theta^{n-1}\left(u_{1} \ldots u_{j}\right)$ occurring in $\omega$ and where $0 \leq i_{l}$, $i, j_{l}, j \leq 3,1 \leq k \leq 4$, and $0 \leq l \leq n-2$. Applying the previous estimate and Lemma 3.3, we obtain

$$
\begin{aligned}
& |h(\omega, a)-h(\omega, b)|=|f(\omega[a, b-1])| \\
& \quad \geq|| f\left(\theta^{n-1}\left(s_{1} \ldots s_{i}\right) \theta^{n}\left(t_{1} \ldots t_{k}\right) \theta^{n-1}\left(u_{1} \ldots u_{j}\right)\right)\left|-2\left(\frac{\sqrt{2} \alpha^{n}}{\alpha-1}\right)\right| \\
& \quad \geq\left(\sqrt{2}-\frac{2 \sqrt{2}}{\alpha-1}\right) \alpha^{n} .
\end{aligned}
$$

Now set $c=\left(\sqrt{2}-\frac{2 \sqrt{2}}{\alpha-1}\right) \approx .2426>0$.

Case 2. No $\theta^{n}(s)$ blocks occur in $\omega[a, b-1]$. Then at least three and at most six $\theta^{n-1}(s)$ blocks occur in $\omega[a, b-1]$. If three or four $\theta^{n-1}(s)$ blocks occur, then the argument used in Case 1 with $n-1$ gives $|h(\omega, a)-h(\omega, b)| \geq$ $c \alpha^{n-1}$.

Otherwise, five or six $\theta^{n-1}(s)$ blocks occur in $\omega[a, b-1]$. Say $\theta^{n-1}\left(s_{1} \ldots s_{i}\right)$ occurs in $\omega[a, b-1]$ for $5 \leq i \leq 6$. But then $s_{1} \ldots s_{i}$ appears in $\theta\left(t_{1} t_{2}\right)$ and $\omega[a, b-1]$ appears in $\theta^{n}\left(t_{1} t_{2}\right)$. In this case, using Lemma 3.1,

$$
\begin{aligned}
|h(\omega, a)-h(\omega, b)| & =|f(\omega[a, b-1])| \\
& \geq|| f\left(\theta^{n}\left(t_{1} t_{2}\right)\right)\left|-3 \max _{s \in S}\right| f\left(\theta^{n-1}(s)\right)|| \\
& \geq\left|2 \alpha^{n}-3\left(2 \alpha^{n-1}\right)\right| \\
& \geq(2 \alpha-6) \alpha^{n-1} \\
& \approx(.8284) \alpha^{n-1} \\
& \geq c \alpha^{n-1}
\end{aligned}
$$

as desired.

Now we can summarize the properties of the cocycle we have constructed:

Theorem 3.2. There exists a uniquely ergodic substitution minimal flow $\left(X_{\omega}, \sigma\right)$ and a cocycle $h: X_{\omega} \times Z \rightarrow R^{2}$ such that

(i) the cocycle $h$ does not grow linearly,

(ii) the cocycles $h_{i}: X_{\omega} \times Z \rightarrow R$ are not covering for $i=1,2$ where

$$
h(x, a)=\left(h_{1}(x, a), h_{2}(x, a)\right),
$$

(iii) the cocycle $h$ is covering. 
Proof. Use Proposition 2.2, Theorem 1.12 of [3], Proposition 2.1, and Theorem 3.1.

Finally, it follows from Proposition 2.2 that some arbitrarily small perturbations of the $h$ we have constructed will have linear growth. However, we do not know how such perturbations effect the structure of the locally compact Hausdorff space $X_{h}$ and the $R^{2}$ action on it.

\section{REFERENCES}

1. F. M. Dekking, Combinatorial and statistical properties of sequences generated by substitutions, Ph.D. Thesis, Katholieke Universiteit te Nijmegen, 1980.

2. _ Recurrent sets, Adv. in Math. 44 (1982), 78-104.

3. H. Furstenberg, H. B. Keynes, N. G. Markley, and M. Sears, Topological properties of $R^{n}$ suspensions and growth properties of $Z^{n}$ cocycles, Proc. London Math. Society (3) 66 (1993), 431-448.

4. W. H. Gottschalk, Substitution minimal sets, Trans. Amer. Math. Soc. 109 (1963), 467-491.

5. B. G. Klein, Homomorphisms of symbolic dynamical systems, Math. Systems Theory 6 (1972), 107-112.

6. K. Madden, The existence and consequences of exotic cocycles, $\mathrm{Ph} . \mathrm{D}$. Thesis, The University of Maryland College Park, 1994.

7. P. Michel, Stricte ergodicité d'ensembles minimaux de substitutions, C. R. Acad. Sci. Paris Sér A 278 (1974), 811-813.

8. M. Queffélec, Substitution dynamical systems-spectral analysis, Lecture Notes in Math., vol. 1294, Springer-Verlag, Berlin and New York, 1987.

Department of Mathematics, Lafayette College, Easton, Pennsylvania 18042

E-mail address: MaddenK@Lafayette.edu 